\title{
GRID SPHERE ELECTRODES USED FOR CURRENT COLLECTION AT THE POSITIVE POLE OF ELECTRODYNAMIC TETHERS
}

\author{
N. H. Stone and J. D. Moore \\ SRS Technologies, 500 Discovery Drive, Huntsville, AL 35806(256)971-7029 \\ N.Stone@stg.srs.com
}

\begin{abstract}
The generation of either electrical power or propulsive thrust with an electrodynamic tether system necessarily depends on driving a return current through the system's ambient space plasma environment. An electrical connection is, therefore, required between the plasma and each end of the tether. The voltage required to drive current through the system is derived either from the orbital motion of the conducting tether through the magnetic field of the Earth, or from a high-voltage power supply that taps into an external energy source (e.g., the sun). In either case, one end of the tether will receive a positive bias. This positive bias, between the tether and the ambient plasma, allows electrons to be collected effectively with a simple, passive electrode. Passive electrode contactors offer several important advantages, including simplification of the upper end-body design and operations, minimization of system mass, and an increase of system reliability and robustness. A preliminary analysis of an inflatable Grid-Sphere end-body concept is presented that is interesting because of the potential for collecting arbitrarily large currents independent of tether length, while the device has the physical characteristics of a high area-to-mass ratio, a low drag coefficient, and simplicity. In particular, we will discuss the physics of current collection by a biased Grid-Sphere and the present state-of-the-art of materials, attainable area-to-mass ratios, and deployment techniques
\end{abstract}

\section{INTRODUCTION}

Electrodynamic tethers use an intriguing application of the Lorentz Force to transform planetary angular momentum into a propulsive force. (When a current is driven from top-to-bottom through a tether that is vertically stabilized by the planetary gravity-gradient, it interacts with the planetary magnetic field to produce a force on the tether in the direction of orbital motion.) When powered by solar energy, these systems require no expellant, thereby offering a "propellantless" thrust capability that is highly desirable for long-duration missions. For example, the MXER momentum-exchange tether concept will, of necessity, rely on electrodynamic tether operations for propellantless re-boost in order to achieve operational efficiencies that are an improvement over competing technologies. Therefore, the efficiency of electrodynamic tether systems, which is the topic of this paper, is of general concern.

In the case of propulsive applications, the operational efficiency of an electrodynamic tether is affected by the energy required to: (1) exchange electrons with the ambient space plasma, and (2) overcome dynamic drag on the system. In addition, the initial cost of launching mass into orbit must be factored into overall system efficiency. The Grid-Sphere concept, assessed in this paper, has been found to offer the po- tential for significant improvements over existing technologies in all three areas.

Passive end-body contactors, which have been validated in space, provide a simple, effective and robust means of current collection at the positive terminal of an electrodynamic tether system. Both the TSS-1 and . TSS-1R missions used a $1.8-\mathrm{m}$ diameter spherical end-body configuration. Moreover, in both cases the current collection efficiency was substantially greater than predicted (requiring only ten percent of the required electrode bias voltage predicted by the best magnetic-limited models of the day) but was limited to a maximum of one ampere by the relatively small collection area. Therefore, to meet the needs of future high-power applications, end-body electrodes must provide a large collection area while maintaining low mass and a low drag coefficient.

Much has been learned about the behavior of tethered systems since TSS that allows the use of less massive end-body architectures. Inflatable, conducting balloon structures can provide a means of obtaining large-scale, very large area-to-mass ratio electrodes that are suitable for electrodynamic tether applications. The mass of a large inflatable end-body will be much less than the ballast mass required to stabilize a tether system during electrodynamic operations [Stone and Gierow, 2001; Stone et al., 2002]. Moreover, balloons have a long space heritage. 
As early as the mid-sixties, inflatable balloons of various sizes and materials, including the "Echo" balloons, which ranged up to $42 \mathrm{~m}$ in diameter, were successfully deployed and tested in space (Goodyear Aerospace Corp., 1982). The prime objective of many of these experiments was to study the radar signature of large bodies. The balloons were, therefore, required to be conductive. This was achieved by laminating a metallic grid between two "photolizable" polybutyl methacrylate films that vanished after 3-4 hours exposure to the space environment (Goodyear Aerospace Corp., 1966, Stimler, 1969). Removal of the films left only a metallic mesh sphere which was 90 percent transparent and, therefore, had a much lower drag coefficient than the original balloon. Drag and gravitational forces were found to be sufficiently low that the mesh structure retained the original spherical inflation geometry of the balloon. Using this technology, a 9.2-m diameter balloon, having a mass of only $9.5 \mathrm{~kg}$, could be packed into a cylinder approximately $30 \mathrm{~cm}$ in diameter $\mathrm{x} 40 \mathrm{~cm}$ long and deployed by a simple inflation mechanism (Goodyear Aerospace Corp., 1982). This early experience with grid spheres may be readily adapted to meet the present requirements of a large-area, passive electrode that is sized to collect large currents and has a high area-to-mass ratio and low drag coefficient.

A Grid-Sphere electrode used for current collection poses additional requirements that did not apply to the previous radar target applications. The electrode must provide the maximum possible cross-sectional area to the influx of electrons while maintaining a low drag coefficient. Considering how the cross sections for neutral and electron fluxes differ provides a means of resolving this apparent dilemma. Two points should be considered:

(1) Dynamic drag in low Earth orbit will result pri- marily from kinetic collisions between neutral particles and the balloon electrode surface (ion densities, typically a factor of $10^{3}$ less than neutrals, are insignificant). Therefore, dynamic drag can be reduced by effectively reducing cross-sectional area for neutrals.

(2) Electrons are hypersonic with respect to the orbital motion and, therefore, the spacecraft, to first order, can be assumed to be stationary with regard to electron motion. The electrons are, however, constrained to move along the geomagnetic field lines, $\mathbf{B}_{0}$. As a result, the electron flux comes primarily from both directions along field lines that intersect the electrode or the inner portion of its plasma sheath (i.e., within an impact parameter).
Because of their high mobility, electrons respond almost instantaneously to electric and magnetic fields and can, therefore, be effectively collected on a b:ased grid. On the other hand, neutitail atoms and molecules are many orders of magnitude more massive than electrons and are not electrically charged. They will, therefore, not respond to the electric field surrounding the grid wires and will pass unimpeded through the grid spacings. The effective transparency for neutrals is simply the optical transparency of the Grid-Sphere, which can be very high-the transparency of the grid material, itself, can be greater than 90 percent. A Grid-Sphere will, therefore, collect electrons while producing minimal neutral dynamic drag-regardless of its orientation to the magnetic field and orbital velocity vectors. No attitude control is required.

The collection of large currents (on the order of ten's of amps) requires a long tether when using present plasma contactor technologies. The collection area of a "bare tether" is directly proportional to tether length, while solid-surface end-body electrodes depend on high voltage to collect large currents because their collection area is limited by drag considerations. A long tether, however, necessarily produces high voltage with its associated hazards, and has a large exposure to the damaging effect of micrometeorite and space debris impacts - both of which are directly proportional to its length. In many applications, tether length must be minimized and high voltage is not desirable. The surface area of the Grid-Sphere electrode, on the other hand, is independent of the tether length and current collection is does not require high voltage. The Grid-Sphere, therefore, may collect large currents without requiring a long tether.

\section{EFFECT OF AN INTERNAL SPACE CHARGE ON CURRENT COLLECTION}

The mass and drag reduction potentials of a GridSphere electrode, discussed above, are quiet apparent and strait-forward-as is the fact that collection area is independent of tether length. However, the current collection efficiency of a grid of $90 \%$ or greater transparency is not obvious. Slightly more than 10 percent of the electron flux to the surface of such a grid would be collected on the initial pass-through. However, as will be shown below, the electrons will generally become trapped within a potential well formed at the surface of the Grid-Sphere and, as a result, will make multiple passes during which most electrons will eventually be collected. 


\section{Calculation of the Internal Space Charge Potential}

The electron flux to the surface of an electrically biased spherical elcetrode immersed in magnetized space plasma is represented, to a good approximation, by the Parker-Murphy model for magneticlimited current collection. This model allows for increased current collection due to the effect of electron gyro-radius expansion produced by the electric field existing in the near vicinity of an electrically biased spherical electrode. The Parker-Murphy model, represented schematically in Figure 1, is given by the simple relation:

$$
\mathrm{I}_{\mathrm{s}}=\mathrm{j}_{\mathrm{o}} \mathrm{A}_{\mathrm{pm}}=\mathrm{j}_{\mathrm{j}}\left(2 \pi \mathrm{R}_{\mathrm{s}}^{2}\right)\left[1+2 \Phi_{\mathrm{s}}^{1 / 2}\left(\frac{\mathrm{R}_{\mathrm{L}}}{\mathrm{R}_{\mathrm{s}}}\right)\right],
$$

where $\mathrm{j}_{\mathrm{o}}=\left(e \mathrm{n}_{\mathrm{o}} \mathrm{v}_{\mathrm{o}} / 4\right), \mathrm{v}_{\mathrm{o}}=\left(2 \mathrm{kT} / \mathrm{m}_{\mathrm{e}}\right)^{12}, \Phi_{\mathrm{s}}=\left(\mathrm{e} \phi_{\mathrm{s}} / \mathrm{kT} \mathrm{T}_{\mathrm{o}}\right)$, $R_{L}=\left(m_{e} v_{d} / B_{o}\right), B_{o}$ is the geomagnetic field, and $R_{s}$ is the radius of the spherical electrode; where $n_{0}$ and $v_{0}$ are the density and thermal velocity of the ambient plasma electrons; where $T_{0}$ and $m_{e}$ are the ambient electronic temperature and mass, respectively, and $k$ is the Boltzmann constant. The Parker-Murphy collection area $A_{\mathrm{pm}}=\left(2 \pi \mathrm{R}_{\mathrm{pm}}{ }^{2}\right)$, is equal to twice the cross-sectional area of a magnetic flux tube of radius $\mathrm{R}_{\mathrm{pm}}$, while the bias voltage, $\phi_{s}$ is taken to be sufficient positive to repel plasma ions.

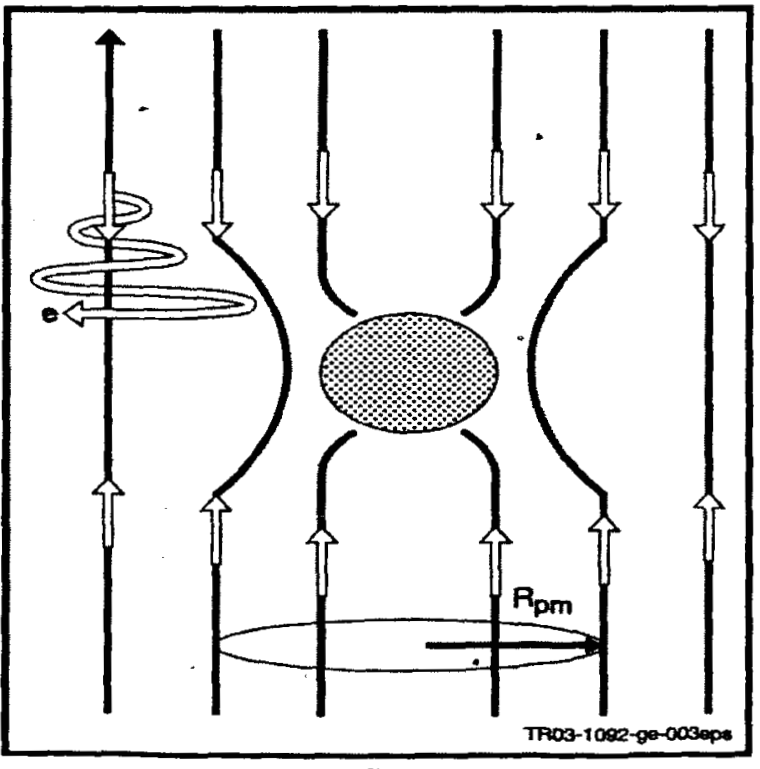

Figure 1. Schematic of the Parker-Murphy magnetically limited current collection model.

Applying the continuity condition to the case of a Grid-Sphere, where the attracted electrons will pass through the surface of the sphere and move into the internal volume, we have, $\left(n v A_{p m}\right)_{o}=(n v A)_{s}=$ (nvA), where the subscripts $o, s$, and $r$ refer to the conditions in the ambient plasma, at the surface of the physical Grid-Sphere of raudius $\mathbf{R}_{\mathrm{s}}$, and at the surface of a virtual sphere of some arbitrary radius $r<$ $R_{s}$, respectively. Using Equation (1), to define $A_{p m}$, leads to:

$$
\begin{aligned}
& n_{\mathrm{o}} v_{\mathrm{o}} {\left[2 \pi R_{s}^{2}\left(1+2 \Phi_{\mathrm{s}}^{1 / 2} \frac{R_{\mathrm{I}}}{R_{s}}\right)\right]=n_{s} v_{s}\left(4 \pi R_{s}^{2}\right) } \\
&=n_{r} v_{r}\left(4 \pi r^{2}\right)
\end{aligned}
$$

Note that the physical and virtual surfaces are spherical while the Parker-Murphy collection surface is twice the area of a circular disc-which accounts for the factor of two differences in the areas. From the first equality of Equation (2) and taking $v_{s} \simeq$ $\left(2 e \phi_{s} / m_{e}\right)^{1 / 2}$, we find the electron density at the surface of the sphere to be

$$
\mathrm{n}_{\mathrm{s}}=\frac{\mathrm{n}_{\mathrm{o}}}{2}\left[\Phi_{\mathrm{s}}^{-1 / 2}+2\left(\frac{\mathrm{R}_{\mathrm{L}}}{\mathrm{R}_{\mathrm{s}}}\right)\right] \text {. }
$$

From the last equality of Equation (2), the density of electrons within the sphere at a radial distance, $r$, from its center is found to be

$\mathrm{n}_{\mathrm{r}}=\mathrm{n}_{\mathrm{s}}\left(\frac{\mathrm{R}_{\mathrm{s}}}{\mathrm{r}^{*}}\right)^{2} \frac{\mathrm{v}_{\mathrm{s}}}{\mathrm{v}_{\mathrm{r}}}$

where $n_{s}$ is given by Equation (3) and $v_{r}$ is a variable yet to be determined. The electron flux to the sphere will, therefore, pass through the grid and create an internal negative space charge, $-\mathrm{en}_{\mathrm{r}}<0$. - As a result, the interior of the Grid-Sphere will not be field-free, as in the case of a simple electrostatics problem. Rather, the resulting space-charge potential will produce an internal radial electric field, $\mathbf{E}(\mathbf{r})$. This field can be found by applying Gauss' law, which states that the electric field vector normal to any closed surface is related to the enclosed charge by the relation:

$$
\oint_{s} \stackrel{r}{\mathrm{E}} \bullet \stackrel{r}{n d a}=\frac{1}{\varepsilon_{0}} \int_{v} \rho d v .
$$

The integral on the LHS of the equation is over the closed surface of a virtual sphere of radius $r \leq R_{s}$. The integral on the RHS is over the volume of the same virtual sphere where $\rho=\rho(r)=-e n(r)$ is the 
charge density distribution. Equation (5) is highly nonlinear and its exact solution is beyond the scope of this paper, but the effect is that electron density will increase with decreasing radius, $r$, resulting in an increasing space charge potential, $\phi(r)$, which will retard the motion of in-flowing electrons. Ultimately, the influx of electrons will be repelled at the value of $r$ where $\left(m_{\mathrm{e}} v_{\mathrm{r}}^{2}\right) / 2=e \phi(r)$. In other words, the electrons that pass through the grid will not pass completely through the sphere and escape from the opposite side. Rather, they will be repelled and possibly trapped within the potential well centered at the GridSphere surface, thereby greatly increasing the collection efficiency.

The behavior of electrons in the vicinity of a biased Grid-Sphere can be shown qualitatively by a simplified treatment. A linearized, first-order solution of Equation (5) for the buildup of the space charge obtained and used to derive a solution for electron velocity in which $\mathrm{V}_{\mathrm{r}}$ is allowed to vary as the space charge $\Phi_{\mathrm{r}}$ grows more negative with decreasing r. From conservation of energy, the incoming electrons will be reflected at a point, $r_{c}$, where their potential energy is equal to the total energy they possessed at the Grid-Sphere surface.

The expression for $r_{c}$ obtained by this approach is

$r_{c}=R_{s} \exp \left[-\frac{n_{0}}{n_{s}}\left(\frac{\lambda_{D}}{R_{s}}\right)^{2} \Phi_{s}\right]$

where $n_{s}$ is given by Equation (3).

Notice that $r_{c}=f\left(n_{0}, \Phi_{s}\right)$. The depth of penetration of the electrons through the surface of the Grid-Sphere is given by

$\mathrm{r}_{\mathrm{p}}\left(\mathrm{n}_{0}, \Phi_{\mathrm{s}}\right)=\mathrm{R}_{\mathrm{s}}-\mathrm{r}_{\mathrm{c}}\left(\mathrm{n}_{\mathrm{o}}, \Phi_{\mathrm{s}}\right)$.

Solution curves of Equation (11) for $\mathrm{r}_{\mathrm{c}}$ as a function of the variables $\Phi_{s}$ and $n_{o}$, are given in Figure $2 a$ for a sphere of radius $R_{s}=8 \mathrm{~m}$; a typical range of ionospheric parameters, $T_{0}=2000 \mathrm{~K}, B_{0}=0.3$ Gauss, and, $n_{0}=10^{8}$ to $10^{12} \mathrm{~m}^{-3}$; and for an applied electrical bias ranging from $\phi_{s}=0$ to 2000 volts. Because of the approximate nature of this solution, a maximum penetration of $r=R_{s} / 10$ is allowed-represented by a horizontal line. The density values used include the

- range typically encountered in low-earth orbitwhich varies from a few times $10^{12} \mathrm{~m}^{-3}$ in sunlight to a few times $10^{10} \mathrm{~m}^{-3}$ in Earth shadow.

The penetration depth, obtained from Equation (12), is shown in Figure $2 b$ and can be seen to increase

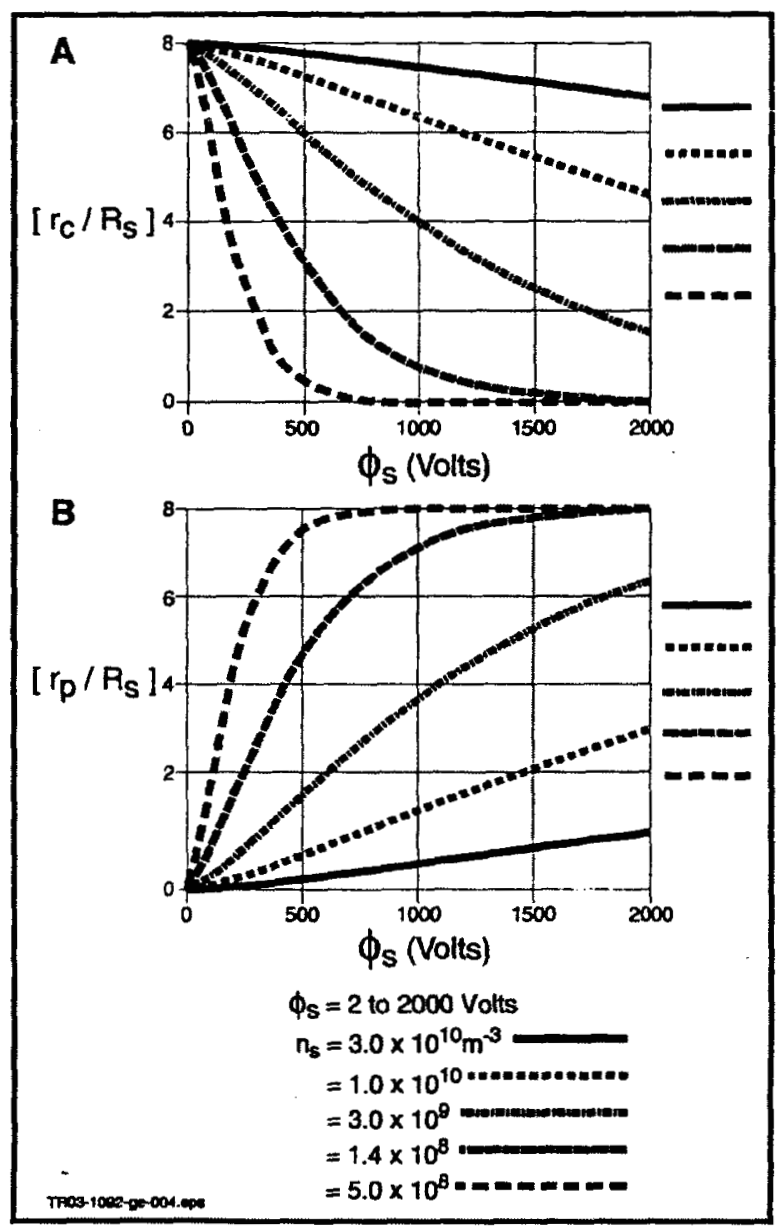

Figure 2. Reflection point for electrons flowing into an 10-m diameter Grid-Sphere as a function of the electric potential imposed on the grid, $\phi_{s}$, for several values of the density of the ambient plasma, $\mathrm{n}_{0}$.

with Grid-Sphere potential and decrease with plasma density. Note that, for $\mathrm{n}_{0}=3 \times 10^{10} \mathrm{~m}^{-3}$ and $\phi_{s} \geq 1000$ volts, and for $\mathrm{n}_{\mathrm{o}}=1 \times 10^{10} \mathrm{~m}^{-3}$ and $\phi_{\mathrm{s}} \geq 400$ volts, $\mathrm{r}_{\mathrm{c}} \leq$ $0.1 R_{s}$. In other words, for these conditions, the electrons that are accelerated toward the Grid-Sphere by the electric field in the sheath will exceed the assumed criteria for maximum penetration depth before being reflected outward by the space potential. However, for potentials less than 400 volts, the electrons will be effectively reflected at all relevant plasma densities.

The important point here is that when electron motion is parallel to $B_{\circ}$ (in the vicinity of the magnetic poles of the Grid-Sphere) the electrons generally will be repelled. In fact, the Grid-Sphere only requires a positive bias of a few ( $>5$ ) volts to reflect the ram atomic oxygen ions. Therefore, a bias of +50 volts will be adequate and, the electrons should never 
penetrate more than a few percent of the Grid-Sphere penetrate more than a few percent of the Grid-Sphere radius in low-earth orbits. Moreover, because of particle collisions (electron-electron and electronneutral) and an imposed azimuthal ExB drift, the reflected electrons, if deflected into non-parallel trajectories, may become trapped within the potential well centered at the Grid-Sphere surface.

\section{Calculation of Electron Trajectories}

The motion of electrons under the influence of the geomagnetic field and the electric field created in the vicinity of the Grid-Sphere is described by

$m \frac{d v}{d t}=-q\left[E_{r}^{r}+\left(v \times B_{0}^{r}\right)\right]$

If we treat only motion in the equatorial plane, then electron velocity, $v$, has components $v_{r}$ and $v_{\varphi}$, which are described by a pair of coupled, first-order differential equations. Solving for the velocities and differentiating the resulting equations with respect to time gives the solution for position in $\mathrm{r}$ and $\varphi$ as functions of time; i.e.,

$$
r(t)=R_{s}+\frac{v_{r o}}{\omega_{c}} \sin \left(\omega_{c} t\right)+\frac{u_{d}}{\omega_{c}}\left[1-\cos \left(\omega_{c} t\right)\right]
$$

and

$$
\varphi(t)=\frac{v_{m}}{\omega_{c}}\left[\cos \left(\omega_{c} t\right)-1\right]+\frac{u_{d}}{\omega_{c}} \sin \left(\omega_{c} t\right)-u_{d} t
$$

where $u_{d}=\left(E_{r} / B_{0}\right)$ and $\omega_{c}=\left(q B_{0} / m\right)$. The electron motion is assumed to be initialized on the surface of the Grid-Sphere at $r_{0}=R_{s}$ with velocity $v_{\mathrm{ro}}=$ $\left(2 \mathrm{e} \phi_{s} / \mathrm{m}\right)^{1 / 2}$, where $\phi_{s}$ is the potential on the GridSphere.

Figure $3 a$ shows a solution of Equations (14a) and (14b) for the conditions: $\theta=0^{\circ}, \alpha=0.90, R_{s}=10 \mathrm{~m}$, $\phi_{s}=500 \mathrm{~V}$, and $n_{0}=10^{10} \mathrm{~m}^{-3}$, where $\theta$ is the angle of the magnetic field to the normal to the equatorial plane and $\alpha$ is the grid transparency. (A large value of $\phi_{s}$ was used simply to make the electron gyrations more visible in the figures.) Figure $3 \mathrm{~b}$ shows the fraction of the electron flux collected as a function of time. Notice that approximately 10 percent is collected on the first pass through the grid. However, because the electrons are trapped in a cyclic motion and gyrate in and out of the Grid-Sphere, they make multiple passes, with additional flux being collected on each subsequent pass. In this case, virtual all of the initial flux is collected within 10 cycles.
Figure 4 shows the effect of increasing the grid transparency to 95 percent (all other parameters are unchanged from the previous case), : hich increases the number of cycles required for total collection to 16 . Figure 5 shows the effect of increasing the angle of the magnetic field from $0^{\circ}$ to $45^{\circ}$ (all other parameters remaining unchanged from the case of Figure 3 ). The increased angle has the effect of weakening the strength of the magnetic field, resulting in a larger diameter gyration which carries the electrons farther around the Grid-Sphere.

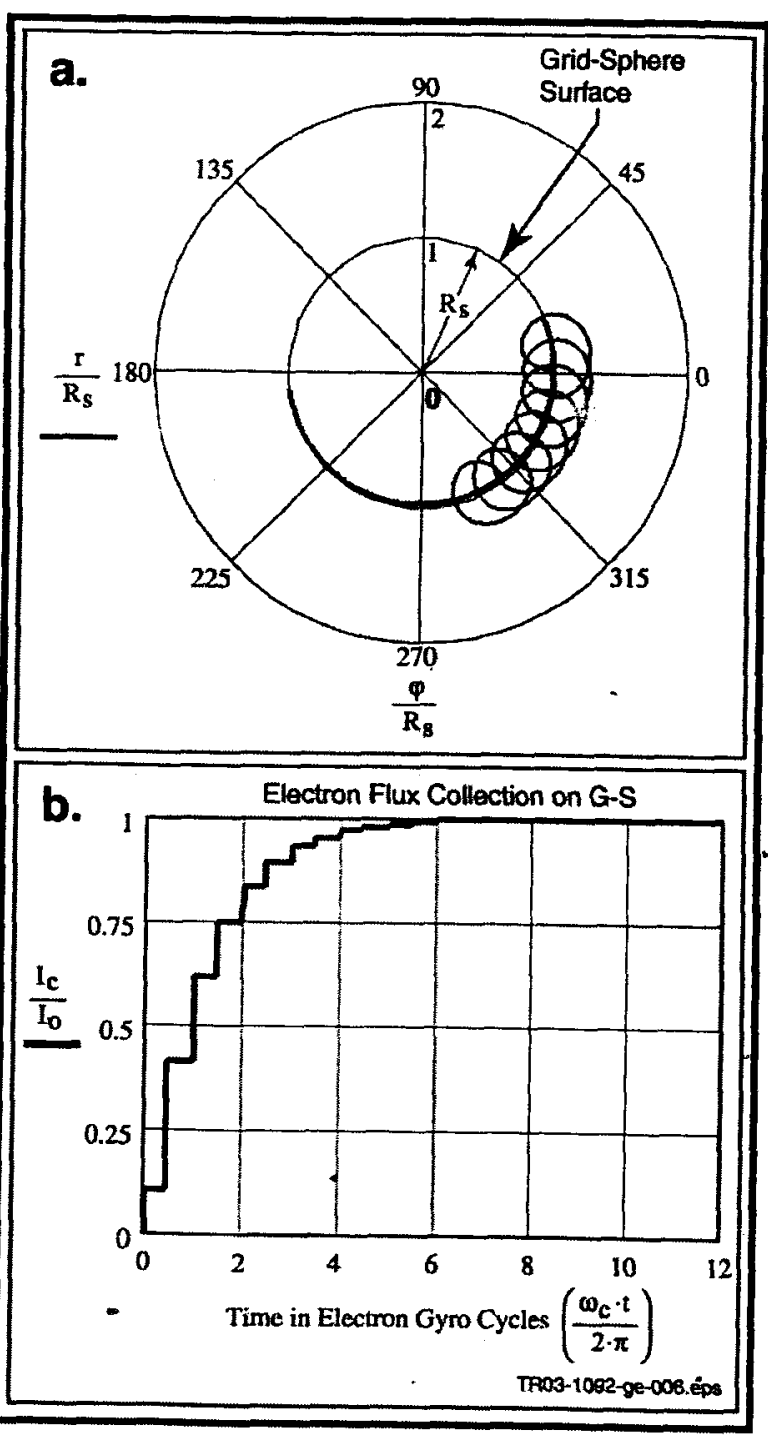

Figure 3. Electron motion in the vicinity of the GridSphere surface showing; (a) Trajectories and cyclic motion of trapped electrons; (b) Collection of electron current with time. The ambient B-field is normal to the $r \varphi$-plane of the Grid-Sphere, which is $90 \%$ optically transparent. 
The above examples show that over most its surface area, the Grid-Sphere will collect near 100 percent of the incident electron flux. In a range of at least $\pm 50^{\circ}$ from its magnetic equator, the collection efficiency will be virtually equal to that of a solid-surface sphere. As discussed in the previous section, the internal space-charge potential will repel electrons that flow along the magnetic field lines near the magnetic poles of the Grid-Sphere, so that they will not be allowed to pass through. However, whether they are trapped and to what efficiency they are collected remain open questions that will require a more exact, three-dimensional collection model.

\section{CONCLUSIONS}

Passive end-body contactors have been validated in space and provide a simple, effective and robust means of current collection at the positive terminal of an electrodynamic tether system. Inflatable, conducting end-bodies, or balloons, offer a technique for obtaining large-area, low-mass electrodes suitable for electrodynamic tether contactor applications. They also have the distinct advantage that the collection area (and, therefore, tether current) is independent of tether length, permitting the design of high-current, short-tether systems.

Neutral-particle drag on an inflatable end-body contactor can be minimized by using a Grid-Sphere

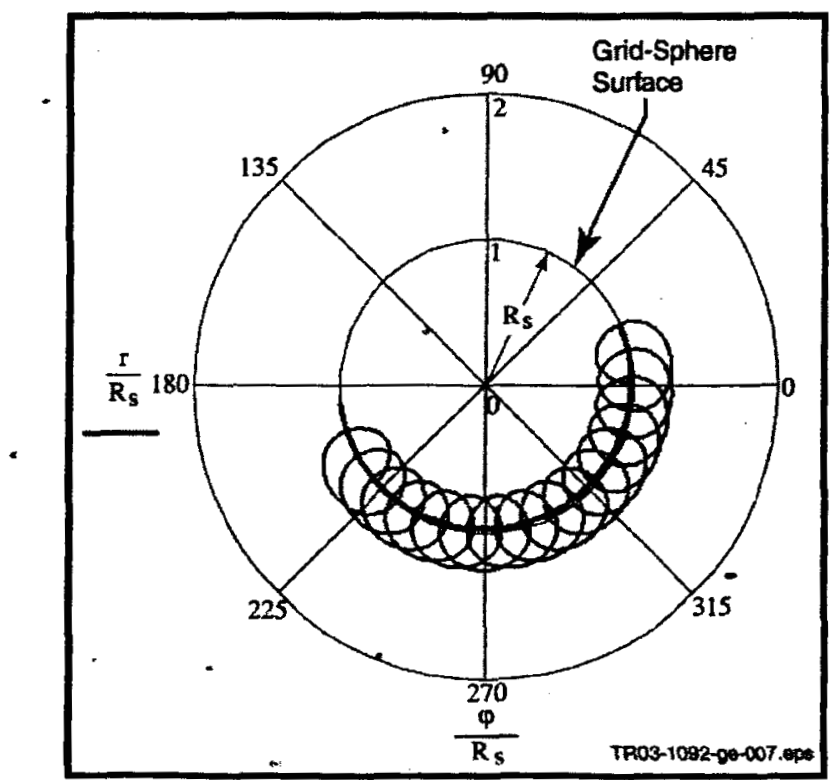

Figure 4. Effect of increased grid transparency on electron motion in the vicinity of the Grid-Sphere surface. Transparency is $95 \%$ compared to $90 \%$ for the case of Figure 3. All other parameters are unchanged.)

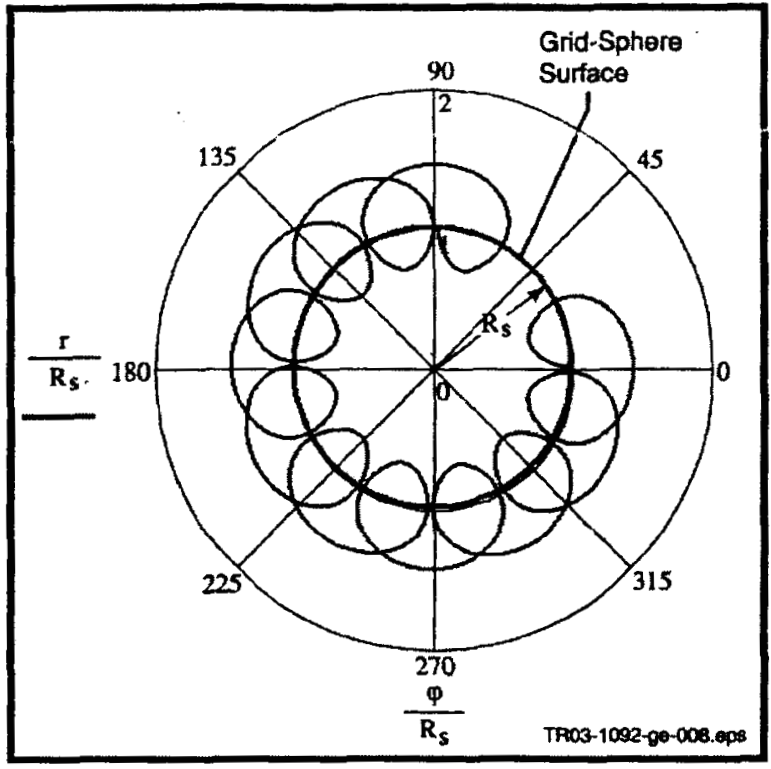

Figure 5. Effect of increased magnetic field inclination on electron motion in the vicinity of the GridSphere surface $\left(45^{\circ}\right.$ compared to $0^{\circ}$ for the case of

Figure 3). All other parameters are unchanged.

rather than a solid surface body. A Grid-Sphere with proper wire spacing will effectively collect electrons, which are prevented from passing through the sphere and are trapped by the combined effect of the internal negative space charge and the geomagnetic field.

It is apparent, even in the Goodyear Aerospace technology from the mid-1940's, that the total GridSphere end-body mass will be less than that typically required to maintain dynamic stability of a tether system during electrodynamic operations. There will, therefore, be no mass penalty associated with the use of inflatable Grid-Sphere electrodes. However numerous advances have been made in thin film materials, fabrication techniques, and deployment technologies since the 1960's that will enhance GridSphere effectiveness. Thin film solar concentrators with large surface areas are have been built by SRS Technologies and flown for satellite solar power augmentation. The concentrators have demonstrated a number of key issues with regard to packaging, deployment, and stability of materials and structures in the space environment. The area-to-mass ratio of the Echo type balloons was about $14 \mathrm{~m}^{2} / \mathrm{kg}$. That of the SRS thin-film solar concentrators is $16.4 \mathrm{~m}^{2} / \mathrm{kg}$, including support structures. SRS has also developed state-of-the-art, large-scale inflatable thin film optical membranes and antennas (see Figure 6). Figure 7 shows a sample of grid-film material recently fabricated by SRS. Unlike the Goodyear Aerospace materials, which sandwiched the metallic grid between two films, this is a single, cast film with an 


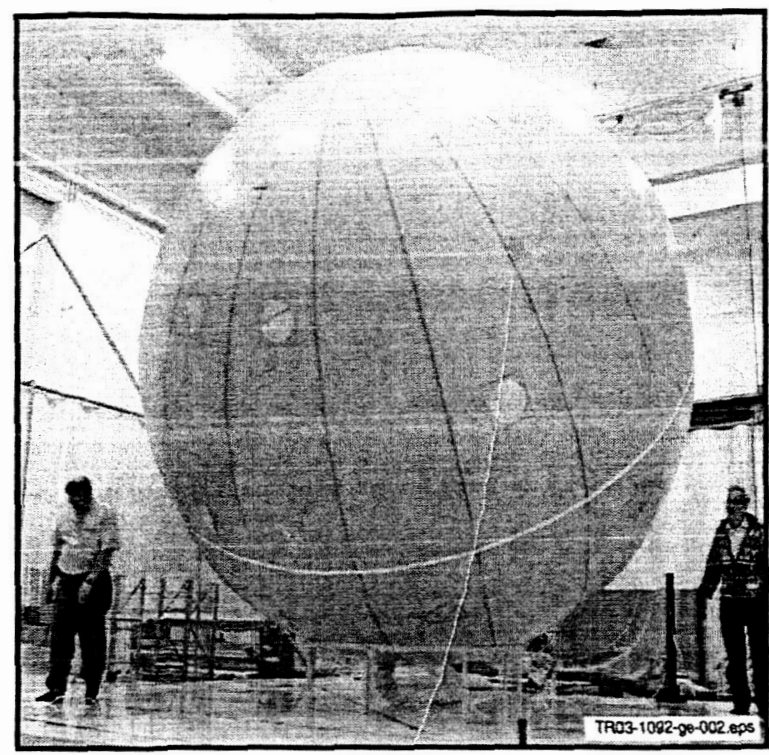

Figure 6. Spherical 5-m diameter inflatable antenna developed by SRS.

embedded grid. With improved film materials and a wation method's available today, it should be possible to produce inflatable spherical end-bodies with very large area-to-mass ratios while maintaining very low drag coefficients.

In summary, the grid-sphere has the distinct advantages of (1) providing virtually any required collection area, and therefore, current-independent of tether length, (2) a low dynamic drag coefficient, (3) a low mass requirement, (4) not requiring attitude control, and (5) a simple and robust, flight-proven deployment mechanism.

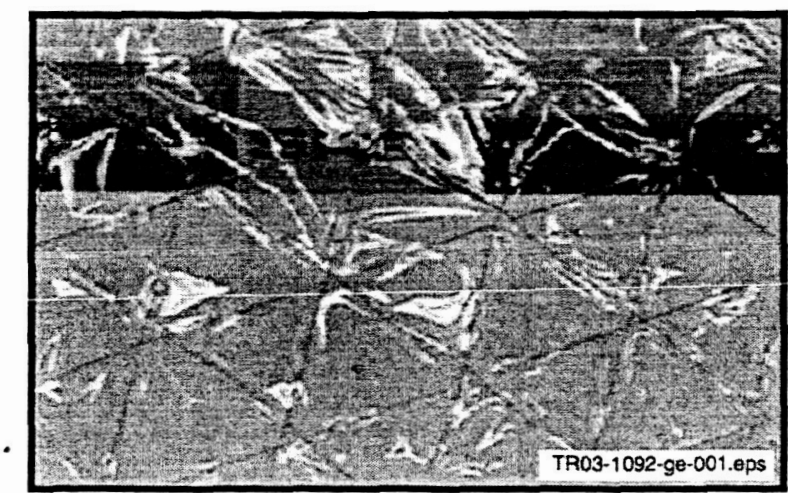

Figure 7. Single-layer, cast grid film material developed by SRS.

\section{ACKNOWLEDGEMENTS}

This work was performed under contract NAS803014 to the NASA Marshall Space Flight Center Advanced Space Transportation Directorate.

\section{REFERENCES}

Goodyear Aerospace Corp., "Orbital experiment of a grid-sphere passive-Communications satellite," Technical Report SEG-TR-66-62, November 1966.

Goodyear Aerospace Corp., "Innovative structures for space applications," GAC 19-1563 Rev A, November 1982.

Khazanov, G.V., N. H. Stone, E. N. Krivorutsky, and M.W. Liemohn, "Current-product field effects on current collection," J. Geophys. Res. 105, 15835 , 2000.

Parker, L. W., and B. L. Murphy, "Proten buildup on an election-emitting ionospheric sattike, $G$. Geophys. Res. 72, 1631, 1967.

Sanmartin, J. R., M. Martinez-Sanchez, and E. Ahedo, "Bare wire anodes for electrodynamic tethers," J. Propul. Power, 9, 353, 1993.

Stimler, F. J., "Grid sphere drag experiment, Goodyear Aerospace Corp." Technical Report AFALTR-69-60, June 1969.

Stone, N, and P. Gierow, "A preliminary assessment of passive end-body plasma contactors," Paper AIAA 2001-1165, 39 ${ }^{\text {th }}$ Aerospace Sciences Meeting; Reno, NV, 8-11 January, 2001.

Stone, N.H., J. D. Moore, W. R. Clayton, and P. A. Gierow, "A preliminary assessment of gridSpheres used as end-body electrodes for electrodynamic tethers," CP608, Space Technology and Applications International Forum-STAIF 2002, (ed) M.S. El-Genk, American Inst. Physics 07354-0052-0/02, Albuquerque, NM, February 2002.

Vannaroni, G., M. Dobrowolny, and F. DeVenuto, "The use of electrodynamic tethers to deorbit LEO spacecraft," IFSI-99-6, Istituto di Fisica dello Spazio Interplanetario, June 1999 